\title{
IDEE E MODELLI DEL PACIFISMO TRA XIX E XX SECOLO
}

di Claudio Giulio Anta

\section{La preziosa bussola di orientamento del dibattito contemporaneo}

Ai fini della stesura di questo saggio, è utile rammentare alcune riflessioni elaborate da prestigiosi intellettuali contemporanei. Norberto Bobbio precisò che il pacifismo poteva agire sui mezzi, sulle istituzioni o sugli uomini. Nel primo caso formulò il concetto di "pacifismo strumentale", la cui azione mirava a limitare gli strumenti di guerra (dottrina e politica del disarmo) o a rifiutare drasticamente il ricorso a comportamenti violenti (la teoria della nonviolenza come il Satyagraha di Gandhi). Invece - puntualizzò ulteriormente Bobbio il "pacifismo istituzionale" poteva essere utilizzato per criticare lo Stato da un duplice di vista. Il primo era riconducibile al "pacifismo giuridico" necessario per costruire uno Stato universale in grado di risolvere i conflitti tra Paesi sovrani. Il secondo era identificabile con il "pacifismo sociale", secondo cui la guerra costituiva un evento derivante da una certa nozione di Stato caratterizzato dalla lotta di classe tra borghesia e proletariato (nelle relazioni interne) e dall'espansione imperialista (nelle relazioni esterne): il rimedio sarebbe consistito nella transizione da una società capitalista a una socialista. Infine, il filosofo torinese delineò il concetto di "pacifismo finalistico" secondo cui la pace poteva essere raggiunta attraverso un' analisi antropologica: la vera ragione della guerra andava ricercata nei difetti morali dell'uomo (Lev Tolstoj) o negli impulsi primitivi della natura umana (Sigmund Freud): a tale proposito, Bobbio utilizzò rispettiva-

Università di Pavia. 
mente le espressioni di "pacifismo etico-religioso" e "pacifismo scientifico"1.

Nella Enciclopedia del Novecento Mulford Quickert Sibley operò una distinzione tra "pacifismo politico" e "pacifismo non politico"2. Il primo enfatizzava le potenzialità dell'attività parlamentare ed era definito anche come "pacifismo della trasformazione", mentre il secondo comportava una limitazione delle esigenze economiche dei cittadini, proponendo loro di vivere in comunità separate dai centri industriali $\mathrm{e}$ dalla vita urbana; in sostanza, secondo Sibley il "pacifismo non politico" implicava "un'etica dell'isolamento e della semplicità". Del resto, tale idea era già stata in parte teorizzata da Lev Tolstoj; infatti, quando il celebre scrittore russo sposò la causa del pacifismo anarchico, concentrò la sua attenzione sulla necessità del lavoro manuale e sul rifiuto di obbedire allo Stato quando esso richiedeva coattivamente ai cittadini il pagamento dei tributi o l'espletamento del servizio militare.

In The New Encyclopcedia Britannica Wilhelm Emil Mühlmann argomentò che il pacifismo poteva essere identificato mediante alcuni principi chiave: il postulato della tolleranza, l'applicazione della dottrina della non-violenza, la limitazione degli armamenti, l'istituzione di tribunali arbitrali neutrali in grado di risolvere le controversie tra $i$ singoli Paesi. Tali presupposti - precisò ulteriormente Mühlmann avrebbero creato le fondamenta per una società etica e armoniosa in grado di perseguire un "integral pacifism" che biasimava la guerra e la violenza come mezzo per risolvere i conflitti in ogni circostanza. Altrettanto ferma fu la sua condanna nei confronti del "semi-pacifism" che, invece, giustificava le guerre in determinati casi; ad esempio, quando esse apparivano "just" o di "defence", oppure contro "unbelievers" o "rebels"3.

Sulla stessa lunghezza d'onda può essere considerato "il pacifismo assoluto" che ebbe tra i suoi sostenitori il filosofo americano

1 N. BoBBIo, Il problema della guerra e le vie della pace, Bologna, il Mulino, 1984, p. 75 sgg..

2 M.Q. SibleY, Pacifismo, in Enciclopedia del Novecento, vol. V, Roma, Istituto della Enciclopedia Italiana fondata da Giovanni Treccani, 1980, pp. 35-47.

3 W.E. Mühlmann, Pacifism and Nonviolent Movements, in The New Encyclopcedia Britannica, Chicago, Benton Foundation and Encyclopaedia Britannica, 1974-1984, vol. 13 , pp. 845-853. 
Michael Allen Fox; egli condannò il concetto di moralità della guerra: "Even military action aimed at protecting people against acute and systematic human-rights violations - sottolineò Fox - [could] not be justified"4. Non distanti apparvero le tesi dello studioso e attivista pacifista statunitense David Cortright, il quale coniò il concetto di "realistic pacifism" per enfatizzare l'assoluta necessità di evitare la guerra nell'era nucleare; sebbene in altri tipi di conflitto - Cortright precisò ulteriormente - "the use of force, constrained by rigorous ethical standards, [might] be necessary at times for self-defense and the protection of the innocent"s.

Quest'ultima riflessione ci permette d'introdurre il concetto di "pacifismo contingente" che ipotizzò la permissibilità, se non addirittura la necessità della guerra in alcuni casi; esso fu sostenuto dal filosofo statunitense Larry May a partire dalla teoria della guerra giusta. A tale proposito, John Rawls ammise "la possibilità di una guerra giusta", ma "non nelle circostanze attuali" considerando il potenziale distruttivo delle armi nucleari7. Non bisogna poi dimenticare la distinzione tra "pacifismo particolare" e "universale": nel primo caso, i pacifisti rivendicarono le loro tesi come meramente personali e, pertanto, non condannarono a priori il ricorso alle armi; al contrario, i pacifisti universali biasimarono incondizionatamente la guerra. A tale proposito, Eric Reitan concepì il pacifismo come "a purely personal commitment to nonviolence"; dunque, esso non poteva essere percepito come "a general obligation to refrain from violence" 8 . Infine Johan Galtung e David Boersema evidenziarono la distinzione tra "negative" e "positive pacifism": il primo era riconducibile alla mera assenza di violenza o guerra, mentre il secondo alla costruzione e consolidamento di relazioni armoniose tra Stati, condizioni necessarie per prevenirla 9 .

4 M.A. Fox, Understanding Peace: A Comprehensive Introduction, New York-London, Routledge, 2014, p. 126.

5 D. Cortright, Peace: A History of Movements and Ideas, Cambridge, Cambridge University Press, 2008, p. 334.

6 L. MaY, Contingent Pacifism: Revisiting Just War Theory, Oxford, Oxford University Press, 2015.

7 J. Rawls, Una teoria della giustizia, Milano, Feltrinelli, 2008, p. 364.

8 E. ReItan, Personally Committed to Nonviolence: Towards a Vindication of Personal Pacifism, in "The Acorn", n. 2, 2000, pp. 30-41 (per la citazione si veda p. 30).

9 J. Galtung, Violence, Peace, and Peace Research, in "The Journal of Peace 


\section{Tra internazionalismo, imperialismo e irrazionalismo}

Le riflessioni di Bobbio, Sibley, Mühlmann, Fox, Cortright, May, Rawls, Reitan, Galtung e Boersema rappresentano un prezioso ancoraggio per comprendere l'evoluzione del pacifismo tra XIX e XX secolo. Traendo inizialmente spunto dalle teorie socialiste, la guerra non era considerata la diretta conseguenza di un particolare tipo di regime politico, bensì di un determinato modo di produzione, vale a dire quello capitalistico; a tale riguardo, Karl Marx e Friedrich Engels formularono la loro teoria della guerra. Solo abolendo la lotta tra la classe lavoratrice e quella capitalistica, sarebbe stato possibile prevenire i conflitti sia all'interno dei singoli Paesi che a livello internazionale; infatti, le guerre erano causate dagli antagonismi tra le borghesie dei vari Stati in competizione tra loro per il controllo dei mercati. La Prima Internazionale elaborò una delle sue più lungimiranti posizioni sui problemi della guerra e della pace durante il Discorso Collettivo adottato al Congresso Internazionale per la Pace di Ginevra (9-12 settembre 1867); in tale occasione, fu dichiarato; "War weighs chiefly on the working class, in that it not only deprives it of the means of existence, but also constrains it to shed the workers'blood"; furthermore, it was stated that "peace, first condition of general well-being, needs in its turn to be consolidated by a new order of things that will no longer know in society two classes, the one of which is exploited by the other"10. Tale approccio ideologico costituì non solo la base su cui il movimento operaio orientò inizialmente la sua azione, ma anche il punto di riferimento per il successivo sviluppo del pensiero marx-engelsiano sulle cause della guerra, vale a dire la teoria dell'imperialismo di Lenin. Ciò avrebbe comportato una revisione della strategia internazionalista e la sua totale avversione alla guerra causata dal sistema degli Stati borghesi. Di fronte a un mas-

Research", n. 3, 1969 pp. 167-191; D. Boersema, Positive and Negative Peace, in A. FialA (ed.), The Routledge Handbook of Pacifism and Nonviolence, New York, Routledge, 2017, chapter 10. La differenza tra pacifismo "negativo" e "positivo" fu sottolineata da Baruch Spinoza (1632-1677) che identificò la pace attraverso la presenza di giustizia, legge e ordine: "La pace non è assenza di guerra: è una virtù, uno stato d'animo, una disposizione alla benevolenza, alla fiducia, alla giustizia". Cfr. B. SpINozA, Trattato teologico-politico, Milano, Adelphi, 1979, vol. I, p. 234.

${ }^{10}$ G.D.H. ColE, A History of Socialist Thought, vol. II, Socialist Thought: Marxism and Anarchism 1850-1890, London-New York, Macmillan-St. Martin's Press, 1954, p. 115. 
sacro che avrebbe coinvolto il proletariato, la causa dei lavoratori idealmente unita da un sentimento di solidarietà - poteva essere perseguita attraverso l'idem sentire pacifista; tuttavia, lo scoppio della Prima guerra mondiale avrebbe causato il fallimento dell'internazionalismo.

Il pacifismo si presentò come antitetico all'imperialismo in quanto basato sulla "ragion di Stato", con particolare riferimento alla dottrina tedesca dello Stato-potenza. Pur non escludendo la pace a priori, l'imperialismo ne minacciava i presupposti poiché prevedeva il primato della politica estera sulla politica interna, nonché l'egemonia militare ed economica dei Paesi più forti rispetto a quelli più deboli (secondo la logica del darwinismo sociale). La diffusione del modello capitalista influenzò profondamente le relazioni internazionali tra XIX e XX secolo; la crescente interdipendenza economica e sociale indusse i Paesi industrializzati ad accaparrarsi nuovi mercati. L'affermazione dell'imperialismo fu analizzata dall'economista inglese John Atkinson Hobson. Nel saggio sull'imperialismo egli confutò la tesi secondo cui le guerre erano generate dalle tendenze aggressive degli esseri umani; infatti, esse non erano il risultato delle "cieche passioni delle razze" o della "follia mista alle ambizioni dei politici" 11 . Invece, le guerre erano generate dai Paesi economicamente più sviluppati che cercavano nuovi mercati al di fuori dei confini nazionali dopo aver raggiunto la soglia di saturazione dei rispettivi profitti. In effetti, la politica estera della Gran Bretagna (che ispirò il lavoro di Hobson) poteva essere inquadrata nell'ambito di "una lotta per [la conquista] dei mercati redditizi"; e ciò riguardava anche la Francia, la Germania, gli Stati Uniti e, più in generale, tutti quei Paesi in cui "il capitalismo moderno [aveva] messo grandi risparmi eccedenti nelle mani di una plutocrazia o di una borghesia risparmiatrice" 12 .

Pur partendo da un diverso contesto storico, tale fenomeno fu analizzato da Vladimir Ilyich Lenin. A suo avviso, il sistema degli Stati borghesi aveva coinvolto il proletariato nella guerra mondiale, poiché non era stato in grado di risolvere le sue contraddizioni. L'analisi di Lenin non differì radicalmente da quella di Hobson; il suo pensiero apparve innovativo, poiché egli identificò l'imperialismo con la fase matura del capitalismo, concretizzatasi quando si "rivelarono i sintomi del

11 J.A. Hobson, L'imperialismo, Milano, Isedi, 1984, p. 44.

12 J.A. Hobson, L'imperialismo, cit., p. 49. 
trapasso a un più elevato ordinamento economico e sociale"13. Tale processo avrebbe condotto verso un "capitalismo di transizione" o, più precisamente, un "capitalismo morente"14; la pace internazionale poteva essere raggiunta solo mediante il superamento di questa fase.

D'altronde già un anno prima della formulazione delle teorie leniniste, il Manifesto adottato dalla Conferenza internazionale dei partiti socialisti di Zimmerwald (5-8 settembre 1915) aveva esplicitamente dichiarato che la guerra rappresentava "il prodotto dell'imperialismo"; vale a dire il tentativo delle classi capitaliste di ogni nazione di alimentare la propria brama di profitto sfruttando il lavoro umano e le risorse naturali. E questa tesi sarebbe stata confermata durante la successiva Conferenza di Kienthal dell'aprile 1916 che ribadì: "The modern development of bourgeois property relations gave rise to imperialist antagonism. The present World War [was] one of the consequences of these antagonisms in the interest of which unsolved national problems, dynastic aspirations, and all the historical relics of feudalism [were] being utilized"15. L'affermazione della politica imperialista costituì uno dei temi più dibattuti anche nell'ambito del Sozialdemokratische Partei Deutschlands (SPD): basti rammentare le tesi sostenute da Karl Liebknecht ${ }^{16}$. Il 2 dicembre 1914 Liebknecht fu l'unico rappresentante della SPD che votò al Reichstag contro il rinnovo dei crediti di guerra. Allo scoppio del primo conflitto mondiale, egli auspicò il risveglio dell'Internazionale socialista, poiché solo attraverso la solidarietà internazionale della classe operaia sarebbe stato possibile arrestare il suo sanguinoso massacro e creare le condizioni per una pace sicura e duratura.

Inoltre, l'ideale della pace dovette confrontarsi con l'irrazionalismo che esaltò la guerra quale fattore di progresso morale o sociale. L'irrazionalismo non fu solo espressione di una crisi di valori, ma anche il terreno preferito di quanti accettarono la realtà senza doversi preoccupare di interpretarla. Tale atteggiamento supino stimolò l'esaltazione

13 V.I. LENIN, L'imperialismo, fase suprema del capitalismo, in V.I. LENIN, Opere complete, Roma, Editori Riuniti, 1966, vol. XXII, p. 265.

${ }^{14}$ V.I. LeNIN, L'imperialismo, fase suprema del capitalismo, cit., p. 301.

15 The Attitude of the Proletariat Towards the Question of Peace (Resolution of the Kienthal Conference), in O. Hess GANKIN, H.H. FisHeR, The Bolsheviks and the World War. The Origins of the Third International, Stanford, Stanford University Press, 1940, p. 421.

${ }^{16} \mathrm{~K}$. Liebknecht, Militarismus und Antimilitarismus, München, Kommunistische Partei Deutschlands, 1907. 
mistica della guerra e, di conseguenza, il riconoscimento del carattere onnipotente dello Stato; l'uomo non doveva comprendere, giudicare o criticare, ma obbedire poiché il senso della storia appariva imperscrutabile. Date tali premesse, si assistette alla nascita di una cultura che in alcuni Paesi (come Germania e Italia) avrebbe giustificato il ricorso alla violenza. Friedrich Nietzsche incarnò in modo emblematico tali valori (o piuttosto disvalori); l'intellettuale tedesco rivalutò l'uomo e la sua "volontà di vivere", negando i valori della civiltà positivista e delineando il concetto dionisiaco della vita, contrario alla metafisica, alla teologia e alla trivialità del quotidiano. Ne scaturì la visione nichilista contenuta in Menschliches, Allzumenschliches (1878), che ribaltò le prospettive del mondo borghese, e il mito della redenzione umana descritto in Jenseits von Gut und Böse (1886) con l'immagine del "Superuomo" condizionato dalla sua "volontà di potenza". Mentre in Morgenröte Gedanken über die moralischen Vorurteile (1881) Nietzsche preconizzò un'epoca di assoluta anarchia per gli uomini: "Per quanto l'utile e la vanità, dei singoli come dei popoli, possano concorrere insieme nella grande politica, l'onda più violenta che li spinge innanzi è il bisogno del sentimento di potenza" che emerge non solo "nelle anime dei principi e dei potenti", ma anche "negli strati inferiori del popolo"17.

Il pensiero nietzschiano suscitò l'attenzione del mondo accademico tedesco, come dimostrò la pubblicazione postuma di Politik (1897-98) di Heinrich von Treitschke, composta da una raccolta di sue lezioni tenute all'Università di Berlino; sostenendo l'idea del pangermanismo attraverso la dottrina dello Stato-potenza, egli scrisse: "Tutti gli Stati a noi conosciuti sono sorti dalla guerra. Perciò essa perdurerà $[\ldots]$ fino a quando esisterà una molteplicità di Stati"18.

Non meno provocatorie apparvero le tesi espresse da Filippo Tommaso Marinetti nel suo Manifesto del Futurismo (1909); l'articolo 9 di tale documento dichiarò che la guerra rappresentava "l'unica igiene del mondo"19. Quindi, essa era identificata come una sorta di purificazione dello spirito umano, il momento ideale per un radicale rinnovamento del-

${ }^{17}$ F. Nietzsche, Aurora. Pensieri sui pregiudizi morali, Milano, Adelphi, 1980, p. 189.

${ }^{18}$ H. von TreitschKe, La politica, Bari, Laterza, 1918, vol. II, p. 2.

${ }^{19}$ Il Manifesto del Futurismo fu pubblicato il 20 febbraio sulla prima pagina de "Le Figaro". 
l'umanità, il terreno fertile per plasmare un nuovo uomo, anche a costo di sacrificare vite umane in nome di un ideale palingenetico. Attraverso una visione apocalittica, Oswald Spengler non lasciò né spazio né speranza per un futuro riscatto della civiltà occidentale ${ }^{20}$. La sua voce non rimase isolata; ad esempio, negli anni Trenta lo scrittore e storico svizzero Louis Gonzague de Reynold descrisse la crisi inquietante di un continente, un tempo dotato di forza civilizzatrice, che ormai aveva perso il suo indiscusso prestigio, mostrando segni di decadenza; al punto tale da evocare l'immagine di una "Europe tragique"21.

\section{Un nuovo ordine europeo tra le guerre mondiali}

Tra il XIX e il XX secolo Ernesto Teodoro Moneta, unico italiano insignito del Premio Nobel per la Pace (1907), occupò una posizione di assoluto rilievo. Dopo aver partecipato alle Cinque giornate di Milano e alla Seconda guerra d'Indipendenza (fu volontario al fianco di Garibaldi tra i "Cacciatori delle Alpi" e nella spedizione dei Mille) ed essersi arruolato nell'esercito del Regno d'Italia rimanendovi fino alla sconfitta di Custoza, egli concluse la carriera militare per dedicarsi al giornalismo che lo avrebbe condotto a una convinta scelta pacifista che affondava le radici nella democrazia risorgimentale d'ispirazione mazziniana. A suo parere, l'indipendenza e la libertà dei popoli non erano tuttavia sufficienti a garantire un assetto pacifico nel Vecchio Continente, poiché sarebbe stato necessario perseguire obiettivi quali il disarmo e l'istituzione di un organo di arbitrato internazionale in grado di dirimere per vie giuridiche le controversie internazionali. Ciò avrebbe creato - scrisse Moneta nell'ottobre 1905 su "La Vita Internazionale" - le premesse per la costruzione di una federazione europea sul modello elvetico, vale a dire una civiltà "senza eserciti armati, e in cui tutte le singole patrie [sarebbero state] confederate in una sola e grande patria comune", riconducibile a quell'"alta meta" di ciascun governo realmente democratico 22 .

20 O. Spengler, Der Untergang des Abendlandes. Umrisse einer Morphologie der Weltgeschichte, 2 vols., Wien-Münich, Braunmüller-Beck, 1918-1923.

${ }^{21}$ L. GonZague de Reynold, L'Europe tragique, Paris, Spes, 1934.

22 E.T. Moneta., L'antimilitarismo nell'esercito, in "La Vita Internazionale", 20 ottobre 1905 , n. 20, pp. 457-459. 
Con l'avvio del primo conflitto mondiale, si affievolì l'idea ereditata dalla filosofia positivista secondo cui la guerra sarebbe scomparsa con l'affermazione delle società industriali. Vi fu anche chi ne evidenziò la sua inutilità economica come il giornalista inglese Norman Angell, autore del pamphlet Europe's Optical Illusion ampliato e pubblicato successivamente con il titolo The Great Illusion. In tale saggio $^{23}$, Angell propose il modello dell'antieconomicità della guerra: in un mondo sempre più condizionato dall' "interdipendenza economica delle nazioni civili”, i conflitti miranti a rafforzare la supremazia politica erano diventati oramai inutili e anacronistici. Angell temeva i rischi di un conflitto, ancor più probabile se gli Stati fossero caduti nella "grande illusione" della tradizionale politica imperialista, nazionalista e colonialista. Le guerre di conquista tra Paesi industrializzati come la Gran Bretagna e la Germania (le relazioni anglo-tedesche erano peggiorate in seguito alle crisi marocchine del 1906 e 1911 e alla rivalità navale) si sarebbero rivelate inutili, poiché il sistema economico internazionale comportava un alto grado d'interdipendenza tra Stati al punto che nessuno di essi avrebbe potuto trarne benefici significativi a spese dell'altro. Le guerre o la conquista di ulteriori colonie si sarebbero rivelate un "errore logico e un'illusione ottica" per i Paesi industrializzati: "La guerra tra le moderne nazioni [doveva] necessariamente fallire al suo scopo: non [poteva] offrire nessun risultato di vantaggio morale o materiale". Ne conseguiva che anche una guerra vittoriosa non avrebbe generato alcun profitto per lo Stato vincitore; dunque, il pacifismo di Angell fu di natura utilitaristica e riconducibile a un "pacifismo particolare" (se utilizziamo la definizione di Reitan).

Le tesi di Angell affondarono le radici in quel pensiero liberale inglese basato sulla logica utilitaristica, che assegnava lo sviluppo armonico e integrato delle relazioni tra Stati alle potenzialità del mercato. Tale idea era già stata sostenuta a metà del XVIII secolo da CharlesLouis de Secondat de Montesquieu in De l'esprit des lois; il filosofo francese aveva osservato: "L'effetto naturale del commercio è di condurre alla pace. Due nazioni che negoziano tra loro diventano reciprocamente dipendenti; se una ha interesse a comprare, l'altra ha interesse

\footnotetext{
${ }^{23}$ N. ANGELL, La grande illusione: studio sulla potenza militare in rapporto alla prosperità delle nazioni, Roma, Voghera Editore, 1913, p. 6.
} 
a vendere; e tutte le unioni sono fondate su reciproci bisogni"'24. E un secolo dopo tale riflessione avrebbe trovato ulteriore riscontro nei Principles of Political Economy di John Stuart Mill: "Il commercio sta rapidamente rendendo obsoleta la guerra, rafforzando e moltiplicando gli interessi personali che sono in naturale opposizione ad essa"25.

Durante il primo conflitto mondiale Romain Rolland evocò invece un'idea di pace idealistica e umanitaria (una sorta di "pacifismo etico" per citare la classificazione iniziale di Bobbio); attraverso la sua vasta produzione letteraria, l'intellettuale francese diffuse un credo di pace e fratellanza, ispirandosi alla rivoluzione russa e alla filosofia orientale (Tolstoj, Gandhi, Gorkij). Quando si rifugiò in Svizzera durante la Grande Guerra, Rolland divenne un punto di riferimento fondamentale per il movimento internazionale pacifista; non a caso il suo instancabile impegno gli valse il premio Nobel per la letteratura (1915). Proprio in Svizzera egli scrisse Au-dessus de la mêlée, pubblicato sul "Journal de Genève" (2223 settembre 1914). Malgrado la forte aspirazione a collocarsi "al di sopra della mischia" per esprimere imparzialità di giudizio nei confronti dei Paesi belligeranti, Rolland manifestò altresì la propria indignazione morale di fronte all'enorme tragedia. Secondo tale autore, la guerra non rappresentava una fatalità e, quindi, un fenomeno inevitabile - tesi sostenuta da Hegel in Grundlinien der Philosophie des Rechts ${ }^{26}$-, ma il risultato della debolezza e della follia del genere umano; più precisamente, il prestigioso scrittore francese definì il conflitto armato in corso - utilizzando un linguaggio incisivo e metaforico - come "une mêlée sacrilège, qui offre le spectacle d'une Europe démente, montant sur le bûcher et se déchirant de ses mains, comme Hercule!"27. Di fronte alla tragicità di un conflitto di così ampie dimensioni, scatenato dalle "trois aigles rapaces" (il Reich tedesco, l'Impero austro-ungarico e la Russia), occorreva creare

${ }^{24}$ C. De SeCondat barone di Montesquieu, Lo spirito delle leggi, Torino, utet, 1996, vol. I, Libro XX, Capo II, Dello spirito del commercio, pp. 528-529.

25 J.S. MILl, Principi di economia politica, Introduzione di G. Becattini, Torino, UTET, 1983, vol. II, p. 800.

${ }^{26}$ G.W.F. HegEL, Lineamenti di filosofia del diritto, con aggiunte compilate da Eduard Gans e note autografe di Hegel, Bari, Laterza, 1965, p. 334.

27 R. Rolland, Au-dessus de la mêlée, in "Journal de Genève", 22 septembre 1914, p. 5. Attraverso il riferimento a Ercole, il letterato francese sembrò preconizzare la "riscoperta" delle Trachinie di Sofocle, tragedia rivalutata a partire dagli Venti da prestigiosi intellettuali quali Henry Prunières, Paul Henri Lang e lo stesso Rolland. 
una "Haute Cour Morale", vale a dire una sorta di "Tribunal des consciences" che vigilasse e giudicasse i crimini perpetrati. E, a testimonianza dell'alto e nobile ideale umanitario di pace e fratellanza che ne contraddistinse il pensiero, Rolland precisò che era necessario elevare lo spirito umano "au-dessus des tempêtes", rimuovendo "les nuages qui cherch [aient] à l'obscurcir". Per poi auspicare, con tinte quasi utopistiche, la costruzione di una "cité [idéale] où s'assembleront les âmes fraternelles et libres du monde entier" 28 , in grado di sconfiggere gli odi nazionali $\mathrm{e}$ l'ingiustizia. Quindi le riflessioni di Rolland sul tema della pace risultarono ideologicamente distanti da quelle di Angell. Il pacifismo di Rolland (come quello di Tolstoj) fu essenzialmente etico e pedagogico, al punto da assumere le caratteristiche di una religione secolare. Al contrario, il pacifismo di Angell apparve utilitaristico, poiché egli non giudicò se la guerra fosse morale o immorale; più pragmaticamente, la considerò un fenomeno superfluo e anacronistico.

Il nuovo ordine europeo delineato dal trattato di Versailles suscitò la convinzione secondo cui il superamento dell'anarchia internazionale rappresentasse la condicio sine qua non per raggiungere una pace duratura; la discussione sulla futura organizzazione del Vecchio Continente fu concomitante alla crisi epocale del sistema degli Stati-nazione d'inizio Novecento che condusse alla creazione della Società delle Nazioni. Proprio l'istituzione ginevrina fu oggetto di un vivace dibattito negli anni Venti; a partire da Richard Nikolaus Coudenhove-Kalergi autore di Pan-Europa e fondatore dell'omonimo movimento, il quale definì la Società delle Nazioni una "anorganische Gliederung"29, poiché non riuniva gli Stati secondo affinità storiche, economiche e culturali, ma in modo meccanico. Ispirandosi alla dottrina enunciata da James Monroe "l'America agli americani”" (formula probabilmente coniata dal suo segretario di Stato John Quincy Adams), CoudenhoveKalergi rivendicò il concetto di "Europa agli europei"; il diplomatico austriaco auspicò un'alleanza di tipo confederale estesa dal Portogallo alla Polonia, distinta dalle altre potenze mondiali quali la Russia comunista e il Commonwealth britannico. Utilizzando come sinonimi i termini di Föderation e Staatenbund, egli si discostò dalle riflessioni

${ }^{28}$ R. Rolland, Au-dessus de la mêlée, cit..

29 R.N. Coudenhove-Kalergi, Pan-Europa, Wien, Pan-Europa Verlag, 1923, p. 83. 
di Alexander Hamilton contenute in The Federalist (1788). Al contrario, Luigi Einaudi auspicò una "seconda specie di Società delle Nazioni", sotto forma di un super-Stato dotato di sovranità diretta sui cittadini, con il diritto di imporre tributi e creare un proprio esercito. Lo statista piemontese delineò una netta distinzione tra i princìpi di "federazione", derivante dall'esempio della Costituzione statunitense, e "confederazione", espressione di una tradizione europea consolidata; la struttura ideata da Thomas W. Wilson - argomentò Einaudi - si collegava al secondo concetto, poiché rappresentava una sorta di alleanza o lega, inadatta a garantire pace duratura ${ }^{30}$. A suo avviso, solo attraverso l'affievolimento della sovranità assoluta degli Stati europei in un'unione federale sarebbe stato possibile superare l'anarchia internazionale e, quindi, evitare nuovi conflitti. In tale ottica, il pensiero di Einaudi può essere idealmente collegato a una forma di "pacifismo giuridico" o "politico" (per rifarsi alle riflessioni di Bobbio e Sibley).

Ad ogni modo, il trattato di Versailles non favorì un processo di riconciliazione tra vincitori e vinti; anzi, suscitò ulteriori motivi di tensione all'interno della comunità internazionale derivanti dal desiderio di mantenere la Germania in uno stato di inferiorità economica e morale. In The Economic Consequences of the Peace John Maynard Keynes biasimò la "Carthaginian Peace of Versailles" 31 ; secondo il prestigioso economista inglese, che partecipò alla Conferenza di pace di Parigi, una politica punitiva avrebbe gettato le basi per una futura guerra. Questo nuovo scenario indusse due liberali britannici, Lord Lothian e Lionel Robbins, a elaborare le proprie tesi federaliste. In Pacifism is not Enough, Lord Lothian (Philip Kerr) cercò di sensibilizzare il movimento pacifista britannico sulle cause della guerra. Egli considerò la pace non solo come una mera "negative condition" in cui la guerra non veniva combattuta, ma anche "a positive thing" o più precisamente "a state of society" in cui le questioni politiche, economiche e sociali erano "settled by constitutional means" 32 . In primo luogo, egli considerò la guerra "inherent" nell' ambito di una scena internazionale dominata dagli Stati sovrani; se-

${ }^{30}$ L. EINAUDI, La guerra e l'unità europea, Milano, Comunità, 1948, pp. 122-123.

31 J.M. Keynes, The Economic Consequence of the Peace, London, Macmillan, 1919, p. 56.

32 Lord Lothian, Pacifism is not Enough, nor Patriotism Either, Oxford, The Clarendon Press, 1935, p. 7. 
condariamente, la Società delle Nazioni così come il Patto Briand-Kellogg non erano strumenti in grado di tutelare "civilisation or peace"; infine, la pace poteva essere garantita solo assicurando "the whole world under the reign of law"33. Lothian confutò altresì la tesi secondo cui il capitalismo e il nazionalismo costituivano le principali cause della guerra: se il capitalismo rappresentava una "international force", poiché gli uomini d'affari raramente manifestavano pregiudizi razziali o nazionali, il nazionalismo era una "creative force" dal momento che stimolava un sentimento basato sulla "common citizenship and common loyalty to the state" piuttosto che su differenze di razza, lingua o religione ${ }^{34}$. A suo parere, il pacifismo e il patriottismo tra le nazioni erano virtù necessarie, ma non sufficienti per costruire una pace duratura; solo una federazione in grado di abbracciare non solo l'Europa, ma il mondo intero avrebbe creato le condizioni necessarie per superare l'anarchia internazionale. In Economic Planning and International Order Robbins evidenziò la crescente interdipendenza economica e sociale prodotta dalla rivoluzione industriale, auspicando un sistema federale che permettesse alle economie di risolvere le crisi grazie alla creazione di sedi di regolazione dei conflitti e programmazione delle priorità economiche: "There must be neither alliance nor complete unification, but Federation"35.

La tesi federalista fu sostenuta non solo da esponenti della scuola inglese, ma anche di quella italiana; negli anni Quaranta essa trovò i principali punti di riferimento nel Manifesto di Ventotene, scritto da Altiero Spinelli ed Ernesto Rossi nel 1941 durante il loro confino politico sull'isola tirrenica e pubblicato nel 1944 da Eugenio Colorni. La federazione europea veniva indicata come l'obiettivo prioritario di un programma politico all'altezza della sfida storica del momento; il superamento della sovranità assoluta degli Stati-nazione costituiva la condizione ritenuta prioritaria per intraprendere qualsiasi iniziativa politica progressista nel continente. Secondo i due intellettuali italiani il nuovo criterio di divisione fra le forze del progresso e della reazione, non era più identificabile mediante "la linea formale della maggiore o minore democrazia, del maggiore o minore socialismo da istituire", ma

${ }^{33}$ Lord Lothian, Pacifism is not Enough, nor Patriotism Either, cit., p. 10.

${ }^{34}$ Lord Lothian, Pacifism is not Enough, nor Patriotism Either, cit., pp. 13-16.

${ }^{35}$ L. RobBIns, Economic Planning and International Order, London, Macmillan, 1937, p. 245. 
con la linea che discriminava "quelli che concepi[vano] come fine essenziale della lotta quello antico, cioè la conquista del potere politico nazionale $[\ldots]$ e quelli che [avrebbero visto] come compito centrale la creazione di un solido Stato internazionale" 36 . In sintesi, i federalisti britannici e italiani s'ispirarono al pensiero hamiltoniano; confrontando le due Costituzioni americane del 1781 e 1787 essi sottolinearono la superiorità del modello federale rispetto a quello confederale, poiché in grado di legalizzare le relazioni tra gli Stati nazionali. Essi sostennero implicitamente un "pacifismo positivo" (per riprendere la classificazione utilizzata da Galtung e Boersema) e altresì un "pacifismo della trasformazione" (si rammenti la definizione di Sibley).

\section{Metodi non violenti al tramonto del secondo millennio}

Anche la diffusione di idee e metodi originariamente estranei alla tradizione democratica occidentale segnò la prima metà del XX secolo nell'ambito del dibattito pacifista. Basti pensare alla teoria della non violenza derivata dagli insegnamenti di Mohandas Karamchand Gandhi, la "Grande Anima" simbolo dell'indipendenza indiana dall'impero britannico. La dottrina gandhiana fu essenzialmente riconducibile alla mistica indù e alla elaborazione di due principi fondamentali: ahimsa e satyagraha. Il concetto di ahimsa stabiliva di non uccidere e mantenere un atteggiamento amichevole; il satyagraha (resistenza passiva) implicava un accordo del proprio essere con la verità e una pratica di condotta morale e sobria. Il tentativo di conformarsi a questi precetti avrebbe comportato uno sforzo interiore per risolvere i problemi dell'esistenza umana con metodi non violenti. Tuttavia, l'ideologia gandhiana affondò le sue radici anche nel pensiero socialista occidentale e in quello utopico; il "Mahatma" fu influenzato da autori quali Henry David Thoreau e, soprattutto, Leo Tolstoj; tant'è vero che nella sua $\mathrm{Au}$ tobiografia Gandhi precisò: "It was [...] when I was passing through a severe crisis of scepticism and doubt that I came across Tolstoy's book, The Kingdom of God is Within You, and was deeply impressed

\footnotetext{
36 A. Spinelli, E. Rossi, Il Manifesto di Ventotene, con un saggio di N. Bobbio, Napoli, Guida, 1982, p. 37.
} 
by it. I was at that time a believer in violence. Its reading cured me of my scepticism and made me a firm believer in ahimsa (nonviolence)" 37 . Il pensiero di Gandhi può dunque essere ricondotto a forme di pacifismo "strumentale", "universale" e "positivo" (per citare i concetti teorizzati rispettivamente da Bobbio, Reitan, Galtung e Boersema).

A partire dalla Prima guerra mondiale Bertrand Russell sostenne con forza la causa pacifista; nei Principles of Social Reconstructions (1916) egli operò una distinzione tra "creative" e "possessive impulses" e, inoltre, precisò: "Liberation of creativeness ought to be the principle of reform both in politics and economics" 38 . I comportamenti umani erano condizionati non solo da desideri consci e diretti, ma altresì da impulsi inconsci e ciechi. Gli impulsi distruttivi che fomentavano la guerra non potevano essere repressi; piuttosto occorreva reindirizzare i derivanti sentimenti di odio e avidità. Russell analizzò ulteriormente le cause psicologiche della guerra nel saggio Political Ideas (1917). Egli evidenziò due diversi tipi di impulsi: da un lato "the possessive ones" riconducibili al desiderio di essere proprietari di beni materiali, limitati e dunque non sempre disponibili; dall'altro "the creative or constructive ones", fondamentali per l'acquisizione della conoscenza umana, che potenzialmente potevano essere sempre soddisfatti. Per ridurre il rischio della guerra le istituzioni politiche avrebbero dovuto valorizzare "the opportunities for the creative impulses" sviluppando, ad esempio, il ruolo dell'educazione, e ridimensionare "the outlets for the possessive instincts" quali cause del ricorso alla violenza e della volontà di dominio ${ }^{39}$.

In tal senso Russell fu interprete di un "pacifismo scientifico" (per rammentare la definizione di Bobbio). In effetti il filosofo gallese sostenne implicitamente le tesi di Sigmund Freud riguardanti il lato oscuro della natura umana; già in due scritti del 1915, Triebe und Triebschicksale ${ }^{40}$ e Zeitgemäßes über Krieg und Tod ${ }^{41}$, il padre della psicoap. 102.

${ }^{37}$ M.K. GANDHI, An Autobiography, Ahmedabad, Navajivan Publishing House, 1969,

${ }^{38}$ B. Russell, The State, in B. Russell, Principles of Social Reconstruction, London, Allen \& Unwin, 1916, p. 6.

${ }^{39}$ B. Russell., Political Ideals, New York, The Century Company, 1917, p. 34.

${ }^{40} \mathrm{Si}$ tratta di uno dei cinque saggi della Metapsicologia. Cfr. S. Freud, Pulsioni e loro destini, in S. FreUd, "Opere”, Torino, Bollati Boringhieri, 1976, vol. VIII, pp. 13-35.

${ }^{41}$ S. Freud., Considerazioni attuali sulla guerra e la morte, in S. Freud, "Opere", Torino, Bollati Boringhieri, 1976, vol. VIII, pp. 119-148. 
nalisi moderna aveva identificato l'aggressività come un impulso intrinseco dell'uomo. Nel saggio Das Ich und das Es (1923) Freud confermò 1'esistenza della "pulsione sessuale" e della "pulsione della morte", evidenziando che dalla loro interazione sarebbe scaturito il precario equilibrio della vita umana ${ }^{42}$. Mentre in Das Unbehagen in der Kultur (1929), egli approfondì nuovamente il legame tra aggressività individuale e guerra: "Homo homini lupus: chi ha il coraggio di contestare questa affermazione dopo tutte le esperienze della vita e della storia?"43. Era sufficiente rammentare le numerose atrocità che avevano contraddistinto la storia umana: dalle invasioni degli Unni o dei Mongoli agli orrori della Grande Guerra, passando attraverso la conquista di Gerusalemme da parte dei Crociati.

Nell'estate del 1932 Freud instaurò una breve ma intensa corrispondenza con Albert Einstein sulla psicologia della guerra. Più precisamente il dibattito avvenne sotto l'egida dell'Istituto Internazionale della Cooperazione Intellettuale, organo consultivo della Società delle Nazioni creato per promuovere lo scambio internazionale tra scienziati, artisti e letterati; la loro corrispondenza sarebbe stata pubblicata nel 1933 con il titolo Why War?44. Il 30 luglio 1932 Einstein scrisse a Freud che la pace poteva essere garantita mediante l'istituzione di un "organo legislativo e giudiziario" internazionale in grado di risolvere le controversie tra Paesi, a condizione che ciascuno di essi ne accettasse senza riserve le decisioni. Si trattava di un obiettivo difficilmente raggiungibile, poiché "la brama di potere" tipica delle classi dirigenti era "ostile a qualsiasi limitazione della sovranità nazionale". Condividendo le opinioni espresse da Freud fin dal 1915, Einstein evidenziò che gli esseri umani covavano potenzialmente una "brama di odio e distruzione"; tali istinti - puntualizzò con implicito riferimento all'oramai definitiva affermazione del regime nazista - esistevano in uno "stato latente", ma tendevano a emergere quando i governanti alimentavano passioni nazionalistiche, imperialistiche e militaristiche. Egli concluse la sua mis-

42 S. Freud., L'Io e l'Es, in S. Freud, “Opere”, Torino, Bollati Boringhieri, 1977, vol. IX, pp. 475-520.

${ }^{43}$ S. Freud., Il disagio della civiltà, in S. Freud, "Opere", Torino, Bollati Boringhieri, 1978, vol. X, pp. 555-630 (la cit. si trova a p. 599).

${ }^{44}$ S. Freud, A. EInStein, Perché la guerra?, in S. Freud, "Opere", Torino, Bollati Boringhieri, 1979, vol. XI, pp. 287-303. 
siva domandando allo psicologo austriaco se era possibile "controllare l'evoluzione mentale dell'uomo" per proteggerlo dalla "psicosi del1'odio e della distruttività" 45 . Nella sua risposta del settembre 1932, Freud concordò con Einstein sulla necessità di una "autorità centrale" alla quale conferire il "diritto di giudicare i conflitti", sostenendo la creazione di una "agenzia suprema" dotata di un reale potere esecutivo. A suo avviso, la Società delle Nazioni non soddisfaceva quest'ultima condizione; infatti, l'istituzione ginevrina appariva eccessivamente condizionata dall' "influenza coercitiva" degli Stati membri che avevano mantenuto la loro sovranità assoluta. Per quanto riguarda i due tipi di istinti umani - "erotici o sessuali" e "aggressivi o distruttivi" Freud precisò che ciascuno di essi era indispensabile, poiché le dinamiche comportamentali degli esseri umani derivavano inevitabilmente dalla loro reciproca interazione, a prescindere che essi "lavorassero di concerto o in contrapposizione". Invece, la guerra poteva essere evitata mediante l'istituzione di una "classe superiore di menti indipendenti" in grado di guidare le masse troppo spesso influenzate dal potere politico e dal condizionamento della Chiesa ${ }^{46}$.

All'indomani della Seconda guerra mondiale il "pacifismo scientifico" di Einstein subì un'evoluzione; a causa della distruttività dell'arma nucleare - dichiarò il padre della relatività - il suo segreto doveva essere affidato a "un governo mondiale" inizialmente composto da Stati Uniti, Gran Bretagna e Unione Sovietica, le tre potenze vincitrici del secondo conflitto mondiale. Nell'ottobre 1947 egli indirizzò una Lettera aperta all'Assemblea generale delle Nazioni Unite; in tale occasione, egli auspicò che l'ONU fosse concepita dai Paesi membri come "a transitional system toward the final goal", vale a dire " $a$ supra-national authority" con "sufficient legislative and executive powers to keep the peace" 47 . In primo luogo, era necessario conferire maggiore potere all'Assemblea generale in modo che il Consiglio di Sicurezza, paralizzato dal potere di veto dei singoli Stati, fosse subor-

${ }^{45}$ Lettera di Albert Einstein a Sigmund Freud, 30 luglio 1932, in S. Freud, A. EINSTEIN, Perché la guerra?, cit., pp. 287-288.

${ }^{46}$ Lettera di Freud a Einstein, settembre 1932, in S. Freud, A. Einstein, Perché la guerra?, cit., p. 289 sgg.

47 A EINSTEIN, Open Letter to the General Assembly of the United Nations, in "United Nations World", October 1947, n. 8, pp. 13-14. 
dinato ad essa; inoltre, occorreva modificare il metodo di rappresentanza nell'ambito delle Nazioni Unite, poiché le procedure di nomina da parte dei governi nazionali non consentivano ai rappresentanti di agire secondo coscienza. In questo modo, l'ONU avrebbe potuto gettare le basi per istituire "a real world government" inizialmente composto da "at least two-thirds of the major industrial and economic areas" del pianeta. E un governo mondiale poteva essere considerato un modello ideale per legalizzare le relazioni tra i vari Paesi, assicurando una pace duratura attraverso l'abolizione della sovranità assoluta dei singoli Stati; attraverso tale idea, Einstein rievocò il contrattualismo di Hobbes in senso kantiano, attribuendogli un valore cosmopolita.

Agli inizi degli anni Cinquanta l'idea del "governo mondiale" animò un dibattito che mirava a delineare l'assetto politico più adeguato di fronte al pericolo di annientamento dell'umanità. Negli Stati Uniti un "Committee to Frame a World Constitution", composto da undici professori universitari, tra cui sei dell'Università di Chicago $0^{48}$, elaborò The Preliminary Draft of a World Constitution (1948). Il Preliminary Draft evidenziava che l'idea del governo mondiale era strettamente connessa al timore di un conflitto nucleare; tant'è vero che la sua istituzione "fondamentalmente e in ultima analisi [era] il problema dell'evo atomico" 49 . L'ordine costituzionale mondiale era concepito dal Committee quale soluzione nei confronti tanto dell'anarchia internazionale quanto della tirannide su scala planetaria; in tale prospettiva, lo "Stato universale" era presentato come la sola alternativa alla distruzione del genere umano. Tuttavia, nel Preliminary Draft emergeva una strategia conservativa nei confronti degli Stati nazionali pur ridimensionati nelle loro funzioni a favore del Governo Mondiale; più precisamente una Costituzione mondiale avrebbe dovuto seguire realisticamente "una via media", cioè prevedere "la sopravvivenza degli stati attualmente esistenti entro una cornice di iniziative e di poteri

${ }^{48}$ Questo Comitato era composto da sei professori dell'Università di Chicago - Robert Maynard Hutchins, Wilber Griffith Katz, Giuseppe Antonio Borgese, Mortimer Adler, Robert Redfield e Rexford Guy Tugwell - quattro professori di altre università americane Stringfellow Barr (St. John's College), Albert Léon Guérard (Università di Stanford), Erich Kahler, (Cornell University) e Charles H. McIlwain (Università di Harvard) - e infine da Harold Innis (Università di Toronto).

${ }^{49}$ Disegno preliminare di costituzione mondiale, Presentazione di P. Calamandrei, Milano, Mondadori, 1949, p. 44. 
locali" e, nello stesso tempo, "spogliare tali stati di funzioni e poteri indispensabili al Governo Mondiale ${ }^{50}$. Jacques Maritain fu tra i principali intellettuali che si fecero interpreti delle proposte formulate dal gruppo di Chicago; attraverso Man and the State (1951) egli auspicò "un'organizzazione autenticamente politica" a livello mondiale, poiché l'umanità si trovava di fronte a un bivio: o il raggiungimento di una pace duratura o il drammatico scenario della guerra atomica. Se il Preliminary Draft aveva analizzato con realismo la sovranità assoluta degli stati nazionali, Maritain la criticò senza mezzi termini: "Bisogna sbarazzarsi del concetto hegeliano o pseudo-hegeliano dello Stato-persona [...] e comprendere che lo Stato non è che una parte e un organo strumentale nel corpo politico" 51 . Dunque, le posizioni del Committee e quelle di Maritain, pur nella loro diversità, rievocarono - per dirla con Cortright - l'idea di un "pacifismo realistico" che sottolineava l'assoluta necessità di evitare la guerra durante l'era nucleare.

D'altronde, la costruzione della bomba all'idrogeno, testata per la prima volta dagli Stati Uniti e dall'Unione Sovietica tra il 1952 e il 1953, suscitò ulteriori timori nella comunità internazionale, poiché causò l'escalation della guerra fredda. In una lettera dell'11 febbraio 1955 Russell propose a Einstein che "six men of the very highest scientific repute", liberi da pregiudizi comunisti o anticomunisti, rendessero "a very solemn statement about the imperative necessity of avoiding war" 52 . Queste furono le premesse che condussero altri nove scienziati a firmare il Manifesto Russell-Einstein che descriveva il potenziale scenario derivante dall'uso della bomba $\mathrm{H}^{53}$. Di fronte alla "tragic situation" gravante sull'umanità - specificò il documento - gli scienziati avrebbero dovuto riunirsi per valutare il pericolo rappresentato dal po-

${ }^{50}$ Disegno preliminare di costituzione mondiale, p. 91. Se ne veda ora la recente edizione pubblicata a Roma, da Edizioni di Storia e Letteratura, nel 2013: G.A. BorgESE, Una costituzione per il mondo, premessa di T. Mann, presentazione di P. Calamandrei, postfazione di S. Bertolotto.

51 J. Maritain, L'uomo e lo Stato, Introduzione di V. Possenti, Genova, Marietti, 2003, pp. 192-193.

52 B. RUSSELL, "In common with every other thinking person", 11 February 1955, Albert Einstein Archives 33-199, Hebrew University of Jerusalem, Jerusalem.

53 I firmatari del Manifesto Russell-Einstein furono gli statunitensi Percy Williams Bridgman, Herman Joseph Muller and Linus Carl Pauling, i britannici Cecil Frank Powell and Joseph Rotblat, il francese Jean Frédéric Joliot-Curie, il polacco Leopold Infeld, il giapponese Hideki Yukawa e il tedesco Max Born. 
tenziale distruttivo della nuova arma e discutere una risoluzione, "not as members of this or that nation, continent or creed, but as human beings, [...] whose existence [was] in doubt" 54 . Durante i primi anni dell'era atomica Einstein fu dunque un sostenitore di un "pacifismo giuridico" (secondo la definizione di Bobbio) attraverso l'idea di un governo mondiale; ma anche di un "pacifismo integrale", "assoluto" e "realistico" (secondo i concetti formulati rispettivamente da Mühlmann, Fox e Cortright) di fronte alla vitale necessità di evitare la guerra nucleare.

In sintesi, i prestigiosi intellettuali che animarono l'intenso dibattito sul pacifismo tra XIX e XX secolo cercarono di incapsulare e risolvere i principali dilemmi del loro tempo: la crisi dell'internazionalismo, l'affermazione dell'imperialismo, la diffusione dell'irrazionalismo, l'inizio della Grande Guerra, l'istituzione e il fallimento della Società delle Nazioni, il consolidamento dei regimi totalitari, lo scoppio della Seconda guerra mondiale, l'origine dell'era atomica e l'escalation della guerra fredda. Malgrado le diverse sfumature di pacifismo progressivamente emerse, essi furono idealmente uniti da un "pacifismo positivo" secondo cui, come predetto da Baruch Spinoza nel suo Trattato teologico-politico e ribadito da Galtung e Boersema nella seconda metà del secolo scorso, la pace non poteva essere concepita come mera assenza di guerra, ma come affermazione di uno status che garantisse giustizia, legge e ordine. Richiamando implicitamente la formula "Pax enim non belli privatio", i protagonisti di tale dibattito hanno così riaffermato un paradigma fondamentale della moderna teoria politica.

\section{Bibliografia ulteriore}

Aldobrandini G., The Wishful Thinking. Storia del pacifismo inglese nell'Ottocento, Roma, Luiss University Press, 2009.

Allan P., Keller A. (eds.), What is a Just Peace?, New York, Oxford University Press, 2006.

Angelini G. (a cura), Nazione, democrazia e pace. Tra Ottocento e Novecento, Milano, FrancoAngeli, 2012.

${ }^{54}$ Il Manifesto del 9 luglio 1955 fu pubblicato su “The New York Times”. 
AnTA C.G., Guerre à la guerre. La leçon de "Coenobium”, Préface par Arturo Colombo, Bruxelles, Peter Lang, 2012.

Atack I., Nonviolence in Political Theory, Edinburgh, Edinburgh University Press, 2012.

Barash D., Webel C., Peace and Conflict Studies, Thousand OaksLondon, Sage, 2002.

Barclay O. (ed.), Pacifism and War, Leicester, Inter-Varsity Press, 1984.

Berlatsky N. (ed.), Pacifism, Farmington Hills, Greenhaven Press, 2011.

Black J. (ed.), The Origins of War in Early Modern Europe, Edinburgh, John Donald Publishers, 1987.

BobBio N., Profilo ideologico del '900, Milano, Garzanti, 1990.

Bonanate L., Guerra e pace: due secoli di storia del pensiero politico, Milano, FrancoAngeli, 1994.

Brock P., Pacifism in Europe to 1914, Princeton, Princeton University Press, 1972.

Brock P., Young N., Pacifism in the Twentieth Century, Syracuse, Syracuse University Press, 1999.

CADy D.L., From Warism to Pacifism: A Moral Continuum, Philadelphia, Temple University Press, 2010.

Carroll B., Flink C., Mohraz J., Peace and War, Santa Barbara, Oxford ABC-Clio, 1983.

Castelli A., The Peace Discourse in Europe, 1900-1945, London, Routledge, 2015.

Cavagnini G., Grossi G. (a cura), Benedetto XV: Papa Giacomo della Chiesa nel mondo dell' "inutile strage", Bologna, il Mulino, 2017.

Ceadel M., Pacifism in Britain: 1914-1945. The Defining of a Faith, Oxford, Clarendon Press, 1980.

Chanteur J., De la guerre à la paix, Paris, PuF, 1989.

ChatField C., For Peace and Justice: Pacifism in America, 1914-1941, Knowille, The University of Tennessee Press, 1971.

Chevalier J., Une dynamique de la paix, Paris, Unesco, 1986.

Clark G., Sohn L., World Peace Through World Law, Cambridge, Harvard University Press, 1958.

Colombo A., Voci e volti dell'Europa. Idee, identità, unificazione, Milano, FrancoAngeli, 2009. 
CoOPer S., Patriotic Pacifism. Waging War on War in Europe 18151914, New York-Oxford, Oxford University Press, 1991.

Defrasne J., Le pacifisme, Paris, PUf, 1983.

DurkeE K., Peace Research: Definitions and Objectives. A Bibliography, Los Angeles, California State University, 1976.

Dyck H.L. (ed.), The Pacifist Impulse in Historical Perspective, Toronto, University of Toronto Press, 1996.

Evans R., Comrades and Sisters: Feminism, Socialism and Pacifism in Europe 1870-1945, Brighton-New York, Wheatsheaf Books-St. Martins, 1987.

FABEI F., La grande guerra e la rivoluzione proletaria. I sindacalisti rivoluzionari dalla neutralità all 'interventismo, Vicenza, In edibus, 2015.

FAUCIER N., Pacifisme et antimilitarisme dans l'entre deux guerres (1919-1939), Paris, Amis de Spartacus, 1983.

Gross E., Das Geheimnis des Pazifismus. Theologie und Politik der Kirchlichen Bruderschaften, Stuttgart, Vorwerk Verlag, 1959.

Grossi V., Le pacifisme européen, 1889-1914, Bruxelles, Bruylant, 1994.

Holmes R.L., Pacifism: A Philosophy of Nonviolence, New York, Bloomsbury, 2016.

HOWARD M., The Invention of Peace: Reflections on War and International Order, Yale, Yale University Press, 2001.

Kaltefleiter W., Pfaltzgraff R. (eds.), The Peace Movements in Europe, London, Croom Helm, 1985.

LipP K., Religiöser Sozialismus und Pazifismus: Der Friedenskampf des. Bundes der Religiösen Sozialisten Deutschlands in der Weimarer Republik, Pfaffenweiler, Centaurus, 1995.

Marchand R., The American Peace Movement and Social Reform, 1898-1918, Princeton, Princeton University Press, 1972.

MARKS S., The Illusion of Peace: International Relations in Europe, 1918-1933, New York, St. Martin, 1976.

Martin D.A., Pacifism, Abingdon, Routledge, 2010.

MerLe M. (dir.), Pacifisme et internationalisme, XVII-XX siècles, Paris, Colin, 1966.

Miller R., Interpretations of Conflict: Ethics, Pacifism and the Just-War Tradition, Chicago, The University of Chicago Press, 1991. 
Mitrany D., A. Working Peace System, Chicago, Quadrangle Books, 1966.

Nelson J.K., The Peace Prophets: American Pacifist Thought, 19191941, Chapel Hill, University of North Carolina Press, 1967.

NutTall G., Christian Pacifism in History, Oxford, Basil Blackwell, 1958.

OpPenheim L., Disputes, War and Neutrality, London, Longmans, 1926.

RAE J., Conscience and Politics: The British Government and the Conscientious Objector to Military Service, 1916-1919, London-New York-Toronto, Oxford University Press, 1970.

Rapoport A., Peace: An Idea Whose Time Has Come, Ann Arbor, University of Michigan Press, 1992.

Reves E., The Anatomy of Peace, New York-London, Harper \& Brothers Publishers, 1945.

Richards L., Christian Pacifism after Two World Wars: A Critical and Constructive Approach to the Problem of World Peace, London, Independent Press, 1948.

SAMPSON R., The Anarchist Basis of Pacifism, London, Stuart Morris Memorial Fund-Peace Pledge Union, 1970.

Santamaria Y., Le pacifisme, une passion française, Paris, Colin, 2005.

Schwartz S.P., A Brief History of Analytic Philosophy: From Russell to Rawls, Hoboken, Wiley-Blackwell, 2012.

Siegel M., The Moral Disarmament of France: Education, Pacifism and Patriotism, 1914-1940, Cambridge, Cambridge University Press, 2004.

Strub J.-D., Grotefeld S., Der gerechte Friede zwischen Pazifismus und gerechtem Krieg: Paradigmen der Friedensethik im Diskurs, Stuttgart, Kohlhammer, 2007.

Trovato S., Mainstreaming Pacifism: Conflict, Success, and Ethics, Lanham, Lexington Books, 2015.

Underhill H., Pacifism: An introductory Perspective, London, Peace Pledge Union, 1991.

VAISSE M. (dir.), Le pacifisme en Europe: des années 1920 aux années 1950, Bruxelles, Bruylant, 1993.

Waltz K.N., Man, the State and War: A Theoretical Analysis, New York, Columbia University, 1959. 
Yoder J.H., The Original Revolution: Essays on Christian Pacifism, Scottsdale, Herald, 1977.

Ziegler D., War, Peace and International Politics, New York, Longman, 2000.

\begin{abstract}
This essay reconstructs the debate on pacifism between the XIX and XX centuries. The author preliminary uses the reflections of some contemporary philosophers and sociologists, such as Norberto Bobbio, Mulford Quickert Sibley, Wilhelm Emil Mühlmann, Michael Allen Fox, David Cortright, Larry May, John Rawls, Eric Reitan, Johan Galtung, and David Boersema. This debate was livened by famous intellectuals: from Karl Marx and Friedrich Engels, to Mohandas Karamchand Gandhi, Bertrand Russell, Sigmund Freud and Albert Einstein passing through John Atkinson Hobson, Vladimir Ilyich Lenin, Friedrich Nietzsche, Norman Angell, Ernesto Teodoro Moneta,
\end{abstract}

Romain Rolland, Luigi Einaudi, Lord Lothian, Lionel Robbins and Jacques Maritain. They encapsulated the main dilemmas derived from the changed political conditions of their time: the crisis of internationalism, the affirmation of imperialism, the spread of irrationalism, the beginning of the Great War, the establishment and failure of the League of Nations, the consolidation of totalitarian regimes, the outbreak of the Second World War, and the escalation of the Cold War. They developed various ideas and models which could ideally be linked to a "positive pacifism" according to which, as foretold by Spinoza, peace could not be conceived as mere absence of war, but above all the presence of justice, law and order. 